\title{
Evaluation of Thyroid Dysfunction in Egyptian Chronic Hepatitis C Virus Cirrhotic Patients Complicated with Portal Hypertension
}

\author{
Waseem M. Seleem MD, Fady M. Wadea MD \\ Internal Medicine Department, Faculty of Medicine, Zagazig University, Zagazig, Egypt.
}

\begin{abstract}
Introduction: Alterations in thyroid hormones regulation and metabolism are observed in cirrhotic patients with decreased serum (T3).Objectives: to investigate the relation of these hormonal alterations to esophageal varices, portal hypertensive gastropathy (PHG) and degree of liver dysfunction in Egyptian cirrhotic patients with portal hypertension. Material and methods: study included 124 cirrhotic patients and 62 controls, patients were stratified into: group I (62 patients) with esophageal varices and group II (62 patients) with portal hypertensive gastropathy without varices, each group was classified according to presence or absence of bleeding into subgroup $A$ and B respectively. Grading of esophageal varices and PHG was done. Serum free T3 (FT3), free T4 and TSH levels were measured using Electrochemiluminescence immunoassay. Results: Cirrhotic patients had significantly lower levels of FT3 than controls (p<0.001). Levels were not significantly differ between bleeders and non bleeders. FT3 was significantly lower in advanced Child's classes and in patients with higher variceal and PHG grades. FT3 was significantly negatively correlated to oesophageal varices, PHG grades and INR. Conclusion: FT3 level was significantly negatively correlated to the degree of hepatic dysfunction, grades of esophageal varices and PHG indicating that FT3 may be used as a non invasive predictor for esophageal varices and PHG grading as well as degree of liver dysfunction in cirrhotic patients with or without bleeding; however, more studies need to be done on a wider scale of patients for confirmation of sensitivity and specificity of adding FT3 to other traditional predictors for varices as splenic size, P.V diameter and platelet count.
\end{abstract}

Keywords: thyroid dysfunction; chronic HCV; esophageal varices; portal hypertension; portal hypertensive gastropathy

\section{Introduction}

Egypt has the highest prevalence of hepatitis $\mathrm{C}$ virus in the world [1]. HCV infection and its complications are major public health problems in Egyptian population [2].

The course of HCV disease varies widely among patients. The symptoms may be either none or only mild and nonspecific. In cases of chronic hepatitis, fibrosis followed by liver cirrhosis may develop in about $10-20 \%$ of patients. In some cases, cirrhosis will end in HCC or life-threatening esophageal and gastric varices [3]

Portal hypertension is abnormally high pressure in the hepatic portal vein. HVPG of $10 \mathrm{~mm} \mathrm{Hg}$ or more is considered as clinically significant portal hypertension [4]. It is manifested by splenomegaly, gastrointestinal bleeding from esophageal varices, gastric varices or portal hypertensive gastropathy (PHG). Ascites, encephalopathy, Caput medusa and internal piles are other manifestations [5]

Liver have an important role in thyroid hormones metabolism which in return play an important role in normal hepatocyte functions. Thyroid dysfunction may occur as an extra-hepatic manifestation of chronic HCV and alterations in thyroid hormone regulation and metabolism are observed in several chronic illnesses including patients with liver cirrhosis [6].

In this study, we aimed to assess the relation of serum thyroid hormones abnormalities to esophageal varices, portal hypertensive gastropathy (PHG) and degree of liver dysfunction in chronic HCV cirrhotic Egyptian patients and studied their correlation to the variceal and PHG grades.

\section{Material and Methods}

This was a hospital based case control study. All subjects of the study were recruited from Gastroenterology and hepatology emergency unit, endoscopy unit and outpatient clinic, Internal Medicine department and the work was conducted in collaboration with Radiology and Clinical Pathology departments, Faculty of Medicine, Zagazig University, Egypt in the period between December 2014 and March 2016.

The study included 124 cirrhotic patients and 62 apparently healthy volunteers as control subjects. The clinical, biochemical, hormonal profiles and radiological investigations of controls were within normal limits.

Severity of the liver disease in cirrhotic patients was classified according to Child's-Pugh classification. All subjects were subjected to complete history taking (including history of previous bleeding episode, upper GIT endoscopy, treatment from thyroid disorders or history of previous operations), thorough full clinical examination and routine biochemical measurement as liver function tests, kidney function tests, $\mathrm{CBC}$, coagulation profile, hepatitis \& autoimmune markers and blood glucose.

\section{Exclusion Criteria}

Patients with other causes of cirrhosis than HCV (as HBV, autoimmune hepatitis,...etc), patients with history of thyroid abnormalities with or without treatments, history of thyroidectomy or drugs affecting thyroid functions especially previous treatment with interferon therapy, diabetic patients, patients with history of variceal injection 


\section{International Journal of Science and Research (IJSR) ISSN (Online): 2319-7064 \\ Index Copernicus Value (2013): 6.14 | Impact Factor (2015): 6.391}

sclerotherapy or band ligation and patients underwent splenectomy with or without vasoligation.

The subjects were divided into 3 groups: (group I) patients who presented with esophageal varices $(n=62)$, (group II) patients who presented with portal hypertensive gastropathy alone without esophageal varices $(n=62)$ and 62 apparent healthy controls (group III). Group I\&II were then classified into subgroups (A and B) according to presence or absence of GIT bleeding respectively. Each subgroup was 31 patients in number.

The ethical committee of Faculty of Medicine at Zagazig University approved our study protocol, a written consent was taken from all patients and control subjects according to Helsinki declaration at recruitment.

\subsection{Estimation of serum TSH, free $T 3$ (FT3) and free T4 (FT4) concentration in patients and controls}

Electrochemiluminescence immunoassay (ECLIA) was performed for in vitro quantitative estimation of TSH, FT3 and FT4 concentrations using COBAS e 411 immunoassay analyzer.

\subsection{Radiological investigations}

A) Pelvi-abdominal ultrasonography using real time grayscale device with a transducer having frequency of 3.5 MHZ. Cirrhotic pattern was determined from the coarse nodular picture of the liver and shrunken size with predominant caudate lobe [7] and portal vein diameter $>13$ $\mathrm{mm}$ indicates portal hypertension [8]

B) Doppler ultrasonography for recording of direction of blood flow in PV, PV patency, maximum velocity and peak flow volume.

\subsection{Upper GIT endoscopy}

Pentax EPK- I 5000 videoscope was used. Esophageal varices were graded according to modified Thakeb classification [9].

Grade I: small and straight varices confined to lower esophageal third.

Grade II: moderate sized clubbed and tortuous varices with normal mucosa in between.

Grade III: large sized varices without apparent normal mucosa in between extending to lower esophageal half.

Grade IV: grade III plus findings of impending variceal rupture (e.g. haemocytes, blue varices and varices on top varices).

Portal hypertensive gastropathy (PHG) was graded according to Tanoue et al. classification [10]:

Grade 0: none.

Grade I: mild PHG

Grade II: moderate PHG.

Grade III: severe PHG.

For patients with portal hypertensive gastropathy bleeding, $32.2 \%$ were presented with manifested GIT bleeding while $67.8 \%$ were presented with iron deficiency anemia and positive occult blood in stool. Patients without bleeding were discovered endoscopically during follow up or at evaluation before starting antiviral therapy.

Regarding cirrhotic patients who didn't bleed; a screening upper GIT endoscopy was performed for detection of esophageal varices and PHG grades in patients who follow the following criteria: ultrasonographic diameter of portal vein diameter $>13 \mathrm{~mm}$ and splenic size $>15 \mathrm{~cm}$ as noninvasive predictor for presence of esophageal varices in patients with liver cirrhosis and portal hypertension [11].

Endoscopic examination was done by 3 endoscopists with intraobserver agreement between them.

\subsection{Statistical analysis}

The obtained data were analyzed statistically using SPSS program version 20. Data were expressed as means \pm standard deviation for quantitative variables and numbers \& percentages for qualitative variables. Independent-Sample (T), (ANOVA), Chi-Square tests $\left(\mathrm{X}^{2}\right)$ and post hoc analysis were used when appropriate. Correlation between variables was done using Person correlation coefficient (r). The results were considered statistically significant if the $P$ value was $<0.05$.

\section{Results}

\subsection{General characteristics of the subjects}

General characteristics of the study subjects are summarized in Table 1. Our subjects were matched as regards age and sex but cirrhotic patients had significantly higher splenic size and portal vein diameter compared to controls $(P<0.001)$. Also patients had GIT bleeding had a higher splenic size and portal vein diameter compared to non bleeders. O.V and PHG grades, gastric varix prevalence and Child's score were significantly higher in bleeders in both group I and II.

\subsection{Thyroid profile levels}

As for serum FT3 levels, we found that cirrhotic patients had significantly lower FT3 concentration compared to healthy controls $(p<0.001)$ (table 2$)$. FT3 levels were not statistically differ between bleeders and non bleeders in either groupI or II. No statistically significant difference between patients and controls as regards FT4 and TSH levels was found $(p>0.05)$ (table 2$)$.

Mean FT3 level was significantly differ among different grades of $\mathrm{O} . \mathrm{V}$ in group I ( $p 0.001$ in group IA and 0.01 in group $I B$ ), post hoc analysis showed that patients had $\mathrm{OV}$ grade III \& IV had statistically significant lower values of FT3 compared to other grades of OV in group IA. Also patients had OV grade II \& III had statistically significant lower values of FT3 compared to grades $0 \mathrm{OV}$ in group IB (Figure 1).

Mean FT3 level was significantly differ between different grades of PHG in group IIB ( $p$ 0.003), post hoc analysis 


\section{International Journal of Science and Research (IJSR) \\ ISSN (Online): 2319-7064 \\ Index Copernicus Value (2013): 6.14 | Impact Factor (2015): 6.391}

shows that there was a statistically significant difference between grade I \&III ( $p$ 0.01) (Figure 2).

On contrary mean FT4 and TSH levels were not significantly differ among different grades of O.V and PHG $(p>0.05)$.

Mean FT3 level was significantly differ among different Child's classes in both groups I\&II, post hoc analysis showed that Child's class (B\&C) had significantly lower FT3 values compared to Child's class (A) in group IA, whereas Child's class (C) patients had significantly lower FT3 values compared to Child's class (A\&B) in group IB. (Figure 3).Also Child's class (C) had significantly lower FT3 values compared to Child's class (B) in group IIA, whereas Child's class (C) patients had significantly lower FT3 values compared to Child's class (A\&B) in group IIB (Figure 4).

Mean FT4 and TSH levels were not significantly differ among different Child's classes in both groups $(p>0.05)$.

\subsection{Correlation of serum thyroid hormones to esophageal varices and PHG grades.}

Serum level of FT3 was significantly negatively correlated to $\mathrm{O} . \mathrm{V}$ grade and INR in group IA ( $p<0.001 \& 0.03)$. In group IB, serum level of FT3 was also significantly negatively correlated to O.V grade $(p<0.001)$, while TSH level was positively correlated to $\mathrm{O} . \mathrm{V}$ grades in group IB (p 0.02) (table 3).

Thyroid hormones levels were not statistically correlated to other parameters as gastric varix, splenic size, portal vein diameter and platelet count $(p>0.05)$ (table 3). Serum FT3 level was significantly negatively correlated to $\mathrm{PHG}$ grades in group IIB ( $p$ 0.02) (table 3).

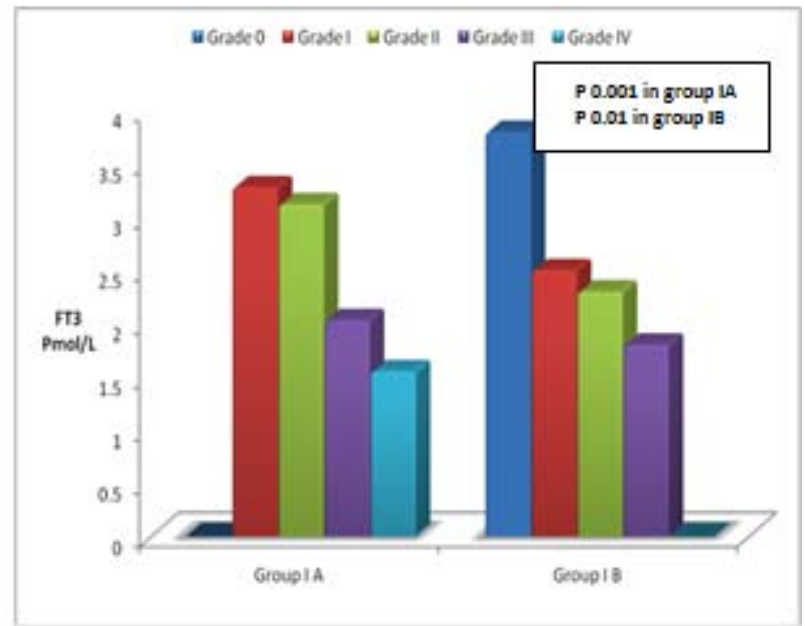

Figure (1) Mean FT3 levels in different O.V grades in group I: significant difference in serum level of FT3 in different OV grades in group I was found; in group IA: significance between grade IV and I\&II was (p 0.02\&0.01 respectively) while significance between grade III and I\&II was (p 0.004, <0.001 respectively). In group IB: significance between grade II and 0 was (p 0.03) while significance between grade III and 0 was ( $\mathrm{p} 0.03$ ).

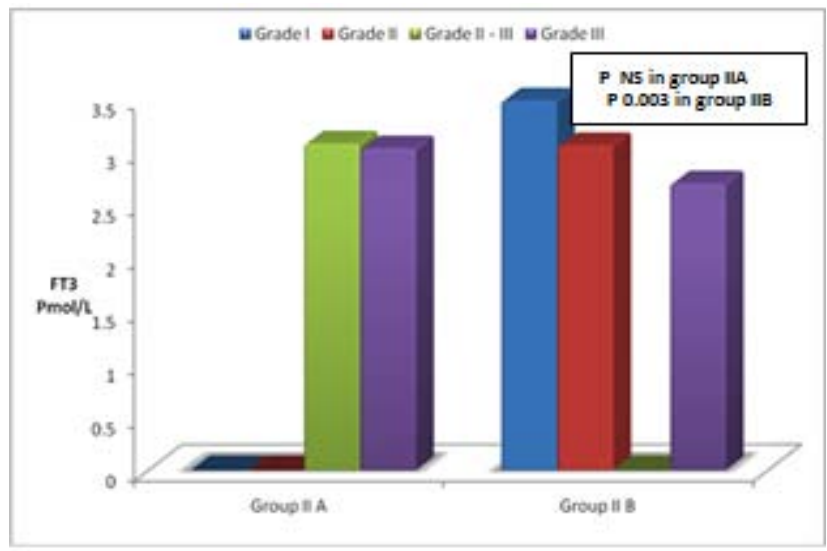

Figure (2) Mean FT3 levels in PHG grades in group II: a significant difference between grade I\&III portal hypertensive gastropathy as regards FT3 levels in group IIB was found ( $\mathrm{p} 0.01$ ) while there was no significant difference between grade II\&III or grade I\&II $(p>0.05)$.

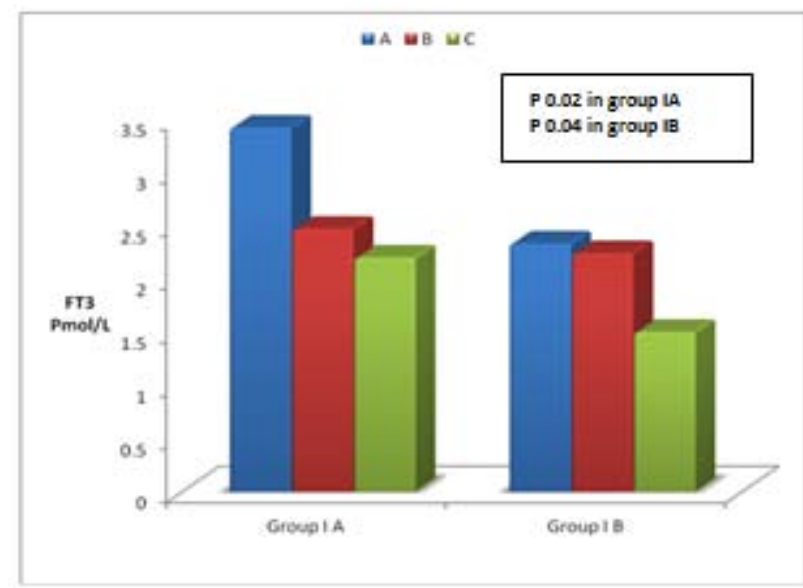

Figure (3) Mean free T3 levels in different Child's classes in group $\mathbf{I}$ : a significant differences as regards serum FT3 levels in different Child's classes in group I was found; in group IA: significance between class B and A was (p 0.03) and significance between class $\mathrm{C}$ and $\mathrm{A}$ was ( $\mathrm{p}$ 0.03). In group IB: Significance between class C and A was (p 0.04) and significance between class C and B was ( $p$ 0.04).

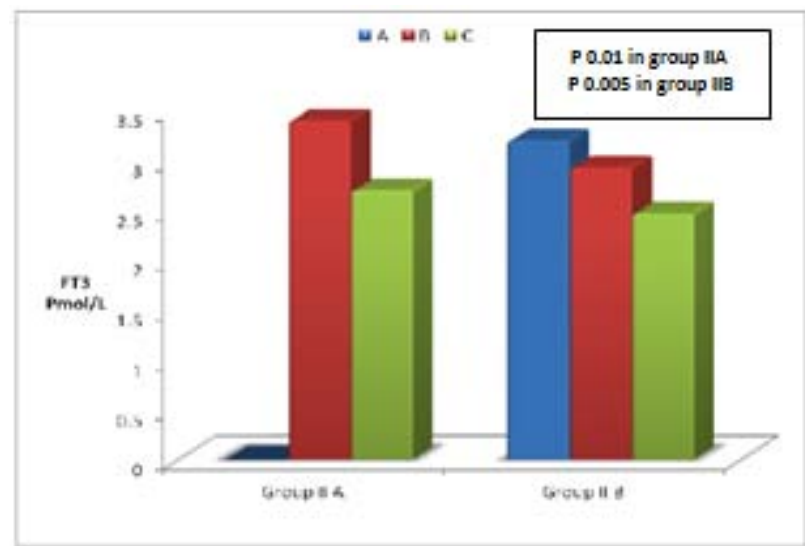

Figure (4) Mean FT3 levels in different Child's classes in group II: significant differences between different Child's classes as regards serum level of FT3in group II was found; in group IIA: significance between Child's class B and C was (p 0.01). In group IIB: significance between class A\&B was (p 0.02), significance between class $A \& C$ was ( $p$ 0.001) while Significance between class B\&C was (p 0.03). 


\section{International Journal of Science and Research (IJSR) \\ ISSN (Online): 2319-7064}

Index Copernicus Value (2013): 6.14 | Impact Factor (2015): 6.391

Table 1: Characteristics of the studied groups

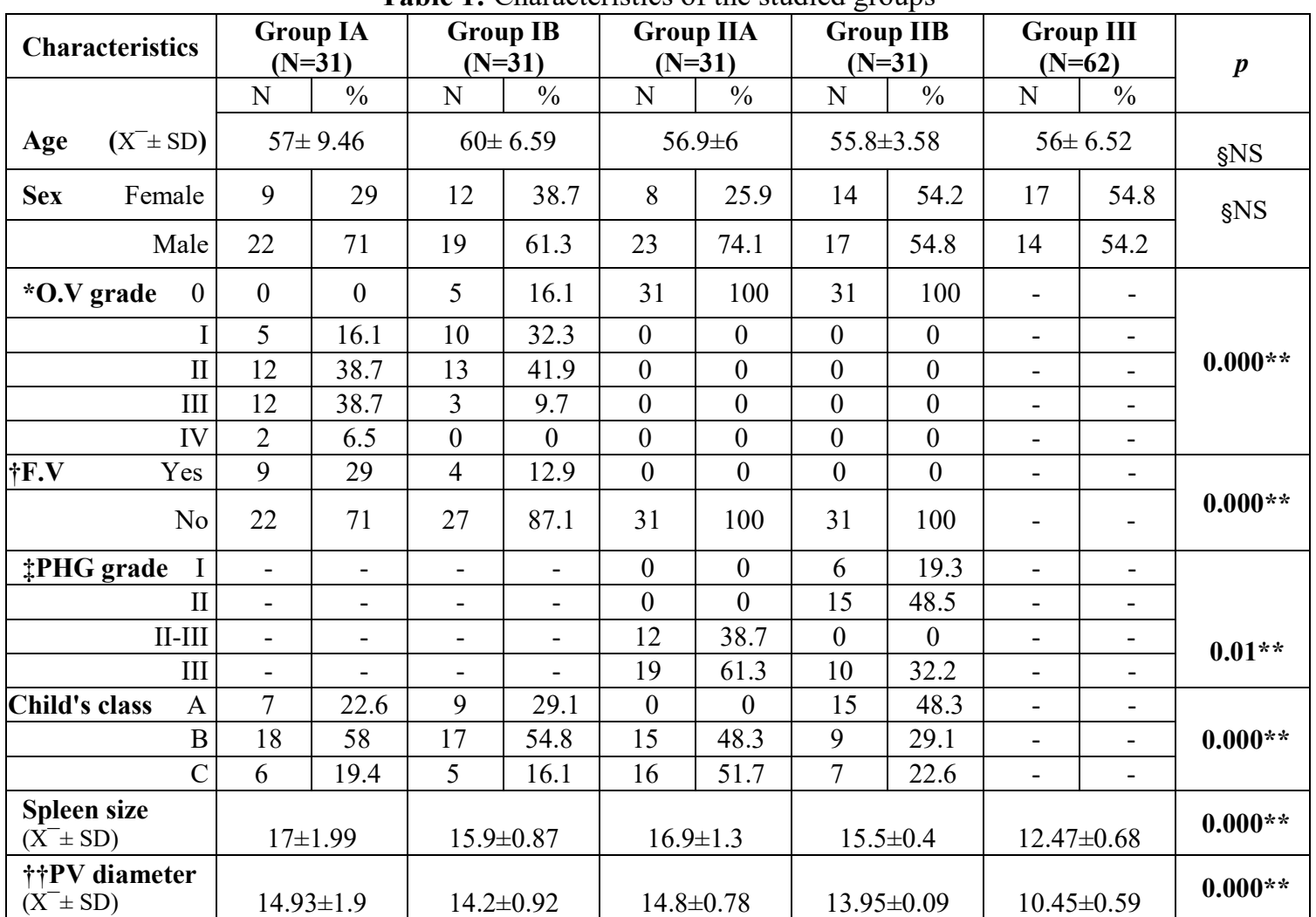

$X^{-}$: mean; SD: standard deviation; ${ }^{*} O V$ : osphageal varices; $\dagger F V$ : fundal varix; $\ddagger P H G$ : portal hypertensive gastropathy; ${ }^{*}$ : significant; ††PV: portal vein; §NS: non significant

Table 2: Thyroid profile among the studied groups

\begin{tabular}{|l|c|c|c|c|c|c|}
\hline Characteristics & $\begin{array}{c}\text { Group IA } \\
(\mathbf{N}=\mathbf{3 1})\end{array}$ & $\begin{array}{c}\text { Group IB } \\
\mathbf{( N = 3 1 )}\end{array}$ & $\begin{array}{c}\text { Group IIA } \\
(\mathbf{N}=\mathbf{3 1})\end{array}$ & $\begin{array}{c}\text { Group IIB } \\
(\mathbf{N}=\mathbf{3 1})\end{array}$ & $\begin{array}{c}\text { Group III } \\
(\mathbf{N}=\mathbf{6 2})\end{array}$ & $\boldsymbol{p}$ \\
\hline TSH & & & & & & \\
\hline$*$ FT3 & $1.22 \pm 1.47$ & $1.41 \pm 1.75$ & $1.57 \pm 0.83$ & $1.19 \pm 0.79$ & $1.70 \pm 1.40$ & $\S \mathrm{NS}$ \\
\hline$\dagger$ FT4 & $2.63 \pm 0.91$ & $2.357 \pm 0.97$ & $3.04 \pm 0.72$ & $3.18 \pm 0.61$ & $4.68 \pm 1.11$ & $\mathbf{0 . 0 0 0 * *}$ \\
\hline
\end{tabular}

${ }^{*}$ FT3: free T3; †FT4: free T4; **: significant; §NS: non significant

Table 3: Correlation between thyroid hormones levels and different parameters in group I\&II

\begin{tabular}{|c|c|c|c|c|c|c|c|c|c|c|c|c|}
\hline & \multicolumn{6}{|c|}{ Group IA } & \multicolumn{6}{|c|}{ Group IB } \\
\hline & \multicolumn{2}{|c|}{ TSH } & \multicolumn{2}{|c|}{ FT3 } & \multicolumn{2}{|c|}{ FT4 } & \multicolumn{2}{|c|}{ TSH } & \multicolumn{2}{|c|}{ FT3 } & \multicolumn{2}{|c|}{ FT4 } \\
\hline & $r$ & $p$ & $r$ & $p$ & $r$ & $p$ & $r$ & $p$ & $r$ & $p$ & $r$ & $p$ \\
\hline $0 . V$ & 0.27 & 0.15 & -0.64 & $0.00 * *$ & -0.25 & 0.18 & 0.40 & $0.02 * *$ & -0.54 & $0.00 * *$ & -0.35 & 0.05 \\
\hline F.V & 0.18 & 0.35 & 0.16 & 0.39 & 0.12 & 0.53 & -0.28 & 0.13 & -0.18 & 0.34 & -0.30 & 0.09 \\
\hline *PLT & -0.04 & 0.82 & 0.31 & 0.09 & 24 & 0.19 & -0.26 & 0.16 & 0.43 & 0.20 & 0.22 & 0.23 \\
\hline INR & -0.13 & 0.49 & -0.38 & $0.03 * *$ & -0.25 & 0.19 & 0.19 & 0.31 & 0.12 & 0.53 & 0.19 & 0.29 \\
\hline Spleen & -0.22 & 0.23 & -0.06 & 0.75 & -0.17 & 0.37 & 0.07 & 0.71 & -0.17 & 0.37 & 0.06 & 0.74 \\
\hline$\dagger P . V$ & 0.04 & 0.82 & -0.28 & 0.13 & -0.30 & 0.09 & -0.01 & 0.96 & -0.1 & 0.59 & 0.1 & 0.98 \\
\hline$\ddagger$ PHG & 0.07 & 0.82 & -0.33 & 0.92 & 0.1 & 0.75 & -0.007 & 0.98 & $\begin{array}{l}-0.69 \\
\end{array}$ & $0.02 * *$ & -0.20 & 0.56 \\
\hline
\end{tabular}

${ }^{*} P L T$ : platelets; $\uparrow P . V$ : portal vein; ${ }^{* *}$ : significant.

$\ddagger$ PHG: portal hypertensive gastropathy correlation to thyroid hormones in group II only.

\section{Discussion}

Liver has a key role in thyroid hormones metabolism in their conjugation, excretion, peripheral deiodination and synthesis of thyroid binding globulin. Thyroid hormones in return have an important role in normal hepatocyte functions by regulation of its basal metabolic rate. Many abnormalities in thyroid gland had been reported in cirrhotic patients include alterations in size of the gland, morphology, architectural pattern as well as alteration of thyroid hormones regulation and metabolism [12].

This study showed significant decrease in FT3 levels in cirrhotic patients compared with controls but no significant differences between groups regarding serum FT4 and TSH levels were present. Our results agree with previous studies which declared that most common thyroid hormones abnormalities reported in cirrhotic patients is low free T3, 


\section{International Journal of Science and Research (IJSR) \\ ISSN (Online): 2319-7064 \\ Index Copernicus Value (2013): 6.14 | Impact Factor (2015): 6.391}

however free T4 and TSH were reported to remain at steady concentrations despite alterations in free T3 level [13].

Low FT3 levels may be due to alterations of two main enzymes which act inside the hepatocytes as part of the iodo-thyronine seleno-deiodinase enzyme system. The type 1 and 3 deiodinases are responsible for extra thyroidal production of $\mathrm{T} 3$ and thyroid hormones inactivation, respectively. The decrease in total $\mathrm{T} 3$ reflects a reduction of deiodinase type 1 activity which results in reduced conversion of T4 to T3 with an increase in conversion of T4 to (rT3) by the deiodinase type 3 system in the liver of patients with cirrhosis [14].

Since the liver is the site of synthesis and catabolism of thyroxin binding globulin (TBG), thyroxin-binding prealbumin (TBPA) and albumin, it is well known that defective hepatic uptake and decreased production of TBG are present in patients with liver cirrhosis. Glucagon levels also affects thyroid hormone concentration and in cirrhotic patients, plasma glucagon concentration is frequently elevated [15]. A significant negative correlation was found between glucagon levels and serum T3 suggesting that increased glucagon level plays a role in the pathogenesis of low T3 concentrations in cirrhotic patients [16].

Steady serum FT4 and TSH levels despite alteration in FT3 concentrations indicates adaptive mechanisms by which the body reduces basal metabolic rate within the liver and this results in preservation of hepatic functions and total body protein storage $[17,18]$.

The study reported significant increase in splenic size and diameter of portal vein in both groups I and II compared with group III. Splenic size and portal vein diameters are usually increased in liver cirrhosis with portal hypertension and could be considered as indicators for esophageal varices presence. Reported splenic size and portal vein diameter associated with esophageal varices development in cirrhotic patients varied from a study to another. Ultrasonographic portal vein diameter $>13 \mathrm{~mm}$ and splenic size $>15 \mathrm{~cm}$ can be considered as non-invasive predictor of esophageal varices in cirrhotic patients with portal hypertension [11]

Our study showed statistically significant relation between Child's class and FT3 levels in both group I and II indicating that serum FT3 concentrations may be considered a sensitive index of hepatic function in liver diseases. These findings are consistent with other studies that suggested that T3 serum levels correlate inversely with the severity of liver dysfunction and could be considered as a good predictor of hepatic dysfunction $[19,20]$. Also, progressive decreases in T3 concentrations in liver disease have been described as indicator of a worse prognosis [21]

In this work we studied the relationship between thyroid hormones levels and esophageal varices and PHG grades. The results showed that FT3 levels differed significantly between different grades of $\mathrm{OV}$ in both group IA and IB Also there were significant negative correlations between FT3 level and OV grades in both group IA and IB.
The grades of esophageal varices often positively correlate with the severity of portal hypertension which correlates with severity of liver disease evaluated according to Child's classification [22] .The severity of liver disease negatively correlates to FT3 level as mentioned before [13] therefore, such mutual correlations may explain why FT3 level decreased in correlation to higher esophageal varices grades.

Similarly we found that FT3 was lower in patients with PHG grade III compared to grade I in group IIB with negative correlation between them, however this correlation was not found in group IIA probably due to the near similarity of PHG grades in studied patients.

It is found that degree of liver dysfunction is correlated with the severity of PHG in patients with cirrhosis [23, 24, 25] and degree of liver dysfunction is negatively correlated to FT3, this explains our finding. Moreover other factor correlated to severity of PHG is previous variceal eradication by repeated endoscopic variceal sclerotherapy or banding $[23,24]$ and because of previous mutual correlation of thyroid dysfunction to esophageal varices described before, this explain why FT3 levels remains low and correlated to severity of PHG following obliteration of the varices with repeated sclerotherapy in some of our patients.

FT3 level was significantly negatively correlated to INR in group of patients presented with variceal bleeding, PT and INR are indicators of severity of liver disease which negatively correlate to FT3 levels and PT is a simple and accurate prognostic marker of liver impairment [26]

Normal or low levels of thyroid-stimulating hormone (TSH) are found in critically ill patient. The changes in serum thyroid hormone levels result from alterations in the peripheral metabolism of the thyroid hormones, TSH regulation, in the thyroid hormone binding to transport protein, in receptor binding and intracellular uptake. Medications in setting of ICU also have a very important role in these alterations [27], this explain why TSH levels remained normal or low in cirrhotic patients presented with GIT bleeding due to different variceal and PHG grades.

No statistically significant correlation between gastric varix and thyroid hormones levels in group I was found which may be attributed to the small number of the patients presented with gastric varix included in this study.

\section{Conclusion and Future Scope}

We can conclude from this observational study that, FT3 level significantly negatively correlated to the degree of hepatic dysfunction and grades of esophageal varices and PHG indicating that FT3 may be used as a non invasive predictor for liver disease status, $\mathrm{OV}$ and $\mathrm{PHG}$ grading in cirrhotic patients with portal hypertension and recommend that thyroid functions should be performed in these patients to detect these changes.

However, more studies need to be done on a wider scale of patients for confirmation of sensitivity and specificity of adding FT3 to other traditional predictors for varices as splenic size, P.V diameter and platelet count for selection of 


\section{International Journal of Science and Research (IJSR) ISSN (Online): 2319-7064 \\ Index Copernicus Value (2013): 6.14 | Impact Factor (2015): 6.391}

cirrhotic patients need a screening upper GIT endoscopic evaluation and also further studies are needed to detect if thyroid hormone supplementations may be of a beneficial effects on these patients or not.

\section{Acknowledgments}

We would like to thank Prof/Dr. Mahmoud Abdou Ashour, Professor of Internal Medicine and Gastroenterology \& Hepatology Unit, Prof. Dr/ Ahmed Gaballa, Professor of Clinical Pathology department, Dr/ Sameh Saber, lecturer of Radiology department and Dr/ Amira Ahmed Abd-elrahman for their generous assistance to complete this work.

\section{Conflict of interest: none.}

\section{References}

[1] Lavanchy D. Evolving epidemiology of hepatitis C virus. Clin Microbiol Infect. 2011; 17(2):107-115.

[2] Mohamoud YA, Mumtaz GR, Riome S, Miller D, AbuRaddad LJ. The epidemiology of hepatitis $C$ virus in Egypt: a systematic review and data synthesis. BMC infectious diseases. 2013; 13(288): 1-21.

[3] Rosen HR. Chronic hepatitis C infection. NEJM. 2011; 364(25):2429-2438

[4] Chang CC, Chen YC, Huang HC, et al. Methimazole alleviates hepatic encephalopathy in bile-duct ligated cirrhotic rats. J Chin Med Assoc. 2006; 69(12):563-568.

[5] Longo DL, Fauci AS, Kasper DL, Hauser SL., Jameson JL, Loscalzo J. Cirrhosis and Its Complications. In Harrison's Principles of Internal Medicine. 2012 18E. Chapter 308: McGraw Hill Professional.

[6] Yan Z, Fan K, Fan Y, et al. Thyroid dysfunction in Chinese patients with chronic hepatitis $\mathrm{C}$ treated with interferon alpha: incidence, long-term outcome and predictive factors. Hepat Mon. 2012; 12(19): e6390.

[7] Dietrich CF, Serra C, Jedrzejczyk M. Ultrasound of the liver. EFSUMB-European Course Book. 2012; P: 31 34.

[8] Al-Nakshabandi, N. The role of ultrasonography in portal hypertension. Saudi J Gastroenterol. 2006; 12(3):111-117.

[9] Thakeb F, Zakaria MS, Hunter M, et al. A study of the esophagus by endoscopy and radiology after sclerotherapy. In Thakeb F. and Zakaria S. (Eds.). Gastrointest Endosc: An Egyptian view, El-Sona ElMohamadia, Egypt1988; 51.

[10] Tanoue K, Hashizume M, Wada H, Ohta M, Kitano S, Sugimachi K. Effects of endoscopic injection sclerotherapy on portal hypertensive gastropathy: a prospective study. Gastrointest Endosc. 1992; 38:582585.

[11] Rani KS, Sudarsi B, Siddeswari R, Manohar S. Correlation of Portal Vein Size with Esophageal Varices Severity in Patients with Cirrhosis of Liver with Portal Hypertension. International Journal of Scientific and Research .2015; 5(1):1-5.

[12] Eshraghian A, Taghavi SA. Systematic Review: Endocrine Abnormalities in Patients with Liver Cirrhosis. Arch Iran Med. 2014; 17(10):713-721.
[13]El-Sawy AAA and Tawfik MA. Low serum free and total tri-iodothyronine hormones as possible prognostic factors in liver cirrhotic patients because of chronic hepatitis C. Tanta Medical Journal. 2015; 43(2):46-51.

[14] Moustafa, AHA, Ali EM, Mohamed, TM and Abdou HI. Oxidative stress and thyroid hormones in patients with liver diseases. Eur J Intern Med. 2009; 20(7):703708.

[15] Smith-Laing G, Orskov H, Gore MBR, Sherlock S. Hypergluconemia in cirrhosis relationship to liver damage. Diabetologia. 1980; 19 (2): 103-108.

[16] Kabadi UM, Kabadi MU, Premachandra BN. Low serum FT3 and raised reverse T3 level in cirrhosis: role of glucagon. Am J Gastroenterol. 1991; 86(10): 15041507.

[17] Ghanaei FM, Mehrdad M, Mortazavi S, Joukar F, Khak M, Atrkar-Roushan Z. Decreased serum total T3 level in hepatitis B and $\mathrm{C}$ related cirrhosis by severity of liver damage. Ann Hepatol.2012; 11(5):667-671.

[18] Kharb S, Garg M, Puri P, Brar KS, Pandit A, Srivastava S. Assessment of thyroid and gonadl function in liver diseases. Indian J Endocrinol Metab. 2015; 19(1):8994.

[19] Shakoor S, Kaneez FS, Iftikhar U. Free T3 as a Reliable Indicator of Thyroid Dysfunction in Cirrhosis. International Journal of Science and Clinical Laboratory. 2012; 7(1):59-72.

[20] Javed R, Ghafoor F, Butt AK, Khan A. Significance of Total and Free Thyroid Hormones in Relation to Serum Proteins in Chronic Hepatitis B Patients and Normal Controls. Significance. 2010; 49(3):85-88.

[21] Guven K, Kelestimur F, Yucesoy M. Thyroid function tests in non-alcoholic cirrhotic patients with hepatic encephalopathy. Eur J Med.1993; 2 (2): 83-85.

[22] Sumon S, Sutradhar S, Chowdhury M, et al. Relation of different grades of esophageal varices with Child-Pugh classes in cirrhosis of liver. Mymensingh Med J. 2013; 22(1):37-41.

[23] Toyonaga A, Iwao T. Portal-hypertensive gastropathy. JGastroenterol Hepatol. 1998; 13:865-877.

[24] Bayraktar Y, Balkanci F, Uzunalimoglu B, et al. Is portal hypertension due to liver cirrhosis a major factor in the development of portal hypertensive gastropathy? Am JGastroenterol. 1996; 91:554-558.

[25] Sarin SK, Sreenivas DV, Lahoti D, Saraya A. Factors influencing development of portal hypertensive gastropathy in patients with portal hypertension. Gastroenterology. 1992; 102:994-9.

[26] Reverter JC. Abnormal hemostasis tests and bleeding in chronic liver disease: are they related? Yes. J Thromb Haemost. 2006; 4:717-20.

[27] Economidou F, Douka E, Tzanela M, Nanas, SA Thyroid function during critical illness. Hormones (Athens). 2011; 10(2):117-24. 